\title{
Percepciones sobre la educación inclusiva del profesorado de una escuela con Programa de Integración Escolar
}

\section{Perceptions of Teachers from a School Integration Program towards Inclusive Education}

\author{
María Elena Mellado Hernández ${ }^{1}$ \\ Universidad Católica de Temuco \\ Teтuco, Chile \\ mmellado@uct.cl \\ Juan Carlos Chaucono Catrinao ${ }^{2}$ \\ Universidad Católica de Temuco. \\ Temuco, Chile \\ juancarchau@yahoo.es \\ Marianela Clementina Hueche Oñate ${ }^{3}$ \\ Universidad Católica de Temuco \\ Temuco, Chile \\ nelly z@gmail.com \\ Omar Andrés Aravena Kennigs ${ }^{4}$ \\ Universidad Católica de Temuco \\ Temuco, Chile \\ omar.aravenak@gmail.com
}

Recibido: 24 octubre 2015 Aceptado: 22 setiembre 2016 Corregido: 22 octubre 2016

\begin{abstract}
Resumen: El objetivo del artículo consiste en develar las percepciones sobre las prácticas de educación inclusiva de las dimensiones pedagógico-didáctica, aprendizaje cooperativo, actuación ética y social que expresan profesionales de un programa de integración escolar (PIE) y docentes de aula, de un centro educativo de la región de Los Ríos, Chile. Este trabajo se suscribe en un estudio de caso, con una metodología mixta de carácter descriptiva. En total participan 36 personas, 15 profesionales de múltiples disciplinas del PIE y 21 docentes de asignaturas del currículo de educación primaria y secundaria. Se les aplicó un cuestionario tipo escala Likert y una entrevista semiestructurada para la
\end{abstract}

1 Académica e Investigadora de la Universidad Católica de Temuco. Doctora en Formación de Profesores e Investigación Educativa de la Universidad de Extremadura, España; Directora del Magíster en Gestión Escolar - Plan de Formación de Directores del Ministerio de Educación, Chile.

2 Docente de educación primaria y director de una escuela de la comuna de Vilcún de la región de La Araucanía, Chile. Magíster en Gestión Escolar de la Universidad Católica de Chile. Estudiante de Doctorado en Innovación en Formación del Profesorado de la Universidad Extremadura, España.

3 Docente de educación secundaria e inspectora general de una escuela de la comuna de Lanco de la región de Los Ríos, Chile.Magíster en Gestión Escolar de la Universidad Católica de Temuco, Chile.

4 Docente de educación media y jefe de la Unidad Técnica Pedagógica de una escuela de la comuna de Río Bueno de la región de Los Ríos, Chile; Magíster en Gestión Escolar de la Universidad Católica de Temuco, Chile; Magíster en Educación Física, Universidad de la Frontera, Temuco, Chile. Estudiante de Doctorado en Innovación en Formación del Profesorado de la Universidad Extremadura, España. 
recogida de información sobre las percepciones de educación inclusiva. Los resultados cuantitativos muestran una leve tendencia hacia planteamientos teóricos asociados a la educación inclusiva. Sin embargo, los hallazgos que surgen del discurso del cuerpo docente se asocian a prácticas excluyentes y segregadas que inhiben el trabajo cooperativo en el aula, lo que demuestra bajas expectativas y una actitud desfavorable hacia la inclusión que provoca desconfianza entre los actores de la comunidad escolar. A modo de conclusión, conviene destacar la necesidad de un currículo inclusivo que promueva lo pedagógico-didáctico y el aprendizaje cooperativo como esencial para una educación inclusiva en el marco de una actuación ética y social, responsable con el desarrollo integral del estudiantado.

Palabras clave: Educación inclusiva, percepción de la educación inclusiva, prácticas de educación inclusiva, programa de escolar, trabajo cooperativo.

\begin{abstract}
The objective of this article is to reveal the perceptions about inclusive education of practices of different professionals from the school Integration Program (PIE) and teachers, considering the pedagogical-didactic dimensions, cooperative learning, ethics and social action in an educational center of Los Rios region, Chile. This work is presented in a case study, using a mixed methodology of descriptive character. 36 people, 15 multidisciplinary professionals from PIE and 21 teachers from different subjects of primary and secondary education, participate in this investigation. A Likert scale questionnaire and a semi-structured interview were applied for collecting information on perceptions of inclusive education. The quantitative results show a slight tendency towards theoretical approaches associated with inclusive education. However, the findings that emerge from the teachers' speech are associated with segregationist and exclusionary practices that inhibit cooperative work in the classroom, showing low expectations and an unfavorable attitude towards inclusion causing distrust among the actors of the school community. In conclusion, it is important to note the need for an inclusive curriculum that promotes pedagogical-didactic and cooperative learning as an essential component for inclusive education within the framework of an ethical and social behavior responsible for the complete development of the student.
\end{abstract}

Keywords: Inclusive Education, Perception of inclusive education, inclusive education practices, School Integration Program, Cooperative work.

\title{
1. Introducción
}

En la última década, la educación inclusiva cobra importancia a nivel mundial, se considera un camino para dar respuesta a la pluralidad y heterogeneidad del estudiantado. En este sentido, la Organización de las Naciones Unidas para la Educación, la Ciencia y la Cultura (UNESCO, 2009) sostiene que el estudiantado tiene derecho a una educación inclusiva que respete las diferencias o dificultades individuales y sociales. Sin embargo, surge como principal problemática el conocimiento y dominio de estrategias efectivas para atender la diversidad en el aula.

En el contexto chileno, el Ministerio de Educación (MINEDUC, 2012) ha impulsado diversos programas en atención a la diversidad y hace esfuerzos por una educación inclusiva con principios y orientaciones para su desarrollo en los centros educativos. Al respecto, UNESCO $(2003 ; 2004)$ declara que resulta imperativo considerar la diversidad de necesidades de todo el alumnado, con el fin de propiciar oportunidades educativas que permitan mejorar la 
participación de los niños y niñas en el desarrollo de sus aprendizajes. En otras palabras, la educación inclusiva promueve una comunidad escolar equitativa, igualitaria y participativa.

Sin embargo, en el sistema educativo chileno persisten políticas que buscan diagnosticar las necesidades educativas especiales (NEE) de cada estudiante para ser considerado en el Programa de Integración Escolar (PIE). Actualmente, existen en Chile cerca de 300.000 estudiantes con NEE que asisten a sala común a clases con docentes de educación primaria y secundaria, según su nivel de enseñanza. Además, tienen apoyo dentro y fuera del aula por un equipo multidisciplinario del PIE (MINEDUC, 2012).

Por otra parte, diversas investigaciones han sostenido la necesidad de un enfoque curricular integrador que propicie un trabajo sistemático y colaborativo entre la comunidad profesional docente, que favorezca el desarrollo integral del estudiantado (Fernández, 2013; González, 2008; Murillo, 2006). Al respecto, López y Blanco (2010) señalan que es preciso avanzar hacia el desarrollo de escuelas más inclusivas que eduquen en la diversidad. En otras palabras, los procesos de enseñanza aprendizaje que se realizan en el aula deberían desarrollarse en torno a las características, diferencias y particularidades de todo el alumnado.

Para lograr en la escuela una práctica inclusiva, es necesario que toda la comunidad escolar (Ferrer y Martínez, 2005), en especial el cuerpo docente, tenga una visión y una actitud positiva acerca de la inclusión. Esta perspectiva compromete al profesorado a implicarse activamente en posibilitar oportunidades de aprendizaje que reconozcan el derecho de aprender que tiene cada estudiante. Esta cultura inclusiva enfrenta el desafío de reestructurar el pensamiento excluyente de docentes que aun sostienen que la atención de determinados alumnos o alumnas puede entorpecer o ralentizar el avance de sus pares.

Es por ello que surge la necesidad de develar las percepciones sobre la educación inclusiva de docentes de aula y de profesionales del Programa de Integración Escolar de un centro educativo, de la región de los Ríos, del sur de Chile.

\section{Referente teórico}

En el mundo educativo todavía se asume como similar el concepto de 'incluir e integrar'. Sin embargo, la inclusión e integración representan enfoques diferentes, aun cuando tengan objetivos aparentemente iguales como la inserción de las personas con discapacidad en la sociedad. Según UNESCO (2003), la integración escolar es un modelo de educación especial que se adapta a las escuelas comunes para integrar al estudiantado que presenta alguna necesidad educativa. En cambio, la educación inclusiva modifica factores del contexto educativo que restringen la participación y desarrollo del aprendizaje de todo el estudiantado. En este sentido, la educación inclusiva reconoce y atiende las necesidades, particularidades y singularidades de cada estudiante y aprende de estas diferencias (Ainscow, 2009; Arnaiz, 2003).

En este sentido, la escuela inclusiva se construye sobre la participación y consenso de toda la comunidad educativa que asume el proceso de aprendizaje del alumnado como la consecuencia de su inclusión en el centro escolar. El propósito de la educación inclusiva es superar las barreras que encuentran algunos alumnos y alumnas desde el inicio de la vida 
escolar (Blanco, 2010; Echeita, 2013; Forteza, 2010). Entonces, la inclusión debería reconocer el derecho que tiene la totalidad de estudiantes a una educación de calidad, cualquiera que sea su medio social, su cultura de origen, su ideología, su etnia, su sexo o su capacidad diferente, ya sea física, intelectual y sensorial, entre otras.

De esta forma, la educación inclusiva está basada en principios de equidad, cooperación y solidaridad y valora las diferencias como oportunidad de enriquecimiento de la sociedad. La inserción es total e incondicional, pues exige rupturas y transformaciones profundas en el sistema escolar (Arnaiz, 2003; Moriña, 2004). En cambio, la integración está centrada en el diagnóstico, en estudiantes con NEE que se derivan a programas específicos, da énfasis a la igualdad y competitividad. En otras palabras, la inserción se centra en el individuo, es parcial y condicionada por resultados estandarizados y da cuenta de comprensiones superficiales sobre la inclusión.

En tal sentido, diversos estudios han consensuado que el profesorado requiere desarrollar estrategias pedagógicas que consideren las características de cada niño y niña (Arnaiz, 2003; Fernández, 2012; Forteza, 2010; Sancho, 2013). Asimismo, Ainscow (2009) plantea que la educación inclusiva promueve un cambio en la cultura escolar que tiene como principal tarea construir una visión inclusiva compartida por toda la comunidad escolar. Dicho de otra forma, se necesita una escuela inclusiva desde una perspectiva más plural que garantice igualdad de oportunidades a todas las personas que participan del centro educativo.

En este contexto, Fernández (2012) plantea cinco dimensiones del proceso inclusivo:

La primera dimensión pedagógico - didáctica, donde cada educador o educadora, de manera individual, debería mantener una actitud positiva ante la diversidad de la comunidad estudiantil, buscando soluciones adecuadas a las necesidades estudiantiles. Es decir, , con el fin de orientar su formación hacia el desarrollo integral de cada niño y niña, el cuerpo docente tendría que asumir un rol mediador entre las características del alumnado, el proceso de enseñanza y la evaluación formativa expresada.

La segunda dimensión corresponde al aprendizaje cooperativo, esta sugiere que el profesorado de aula trabaje en conjunto con el equipo de integración educativa. De tal forma, Durán y Giné (2011) sostienen la necesidad de asumir métodos cooperativos de aprendizaje entre la comunidad profesional. En otras palabras, es indispensable asumir un trabajo cooperativo efectivo con metas comunes entre profesionales del PIE y equipo docente, utilizando como estrategia diálogos reflexivos para evaluar en conjunto la pertinencia de las actividades de aprendizaje según las características y necesidades del cuerpo estudiantil.

La tercera dimensión de actuación ética se considera fundamental para desarrollar un efecto positivo en los aprendizajes. En este marco, Arnaiz (2011; 2012) sostiene que el profesorado que asume una actitud positiva frente a estudiantes que presentan necesidades educativas especiales, es más comprometido con sus tareas y deberes pedagógicos. En otras palabras, la ética profesional favorece una actitud proactiva y de responsabilidad con el aprendizaje de todos los niños y niñas.

Finalmente, la dimensión social establece la necesidad de que exista una relación de confianza entre estudiante, su familia y el equipo docente. Así la comunidad escolar 
asumiría con determinación, de manera conjunta, los desafíos y apoyos curriculares que necesita el grupo estudiantil que presenta NEE. Al respecto, Fernández (2012) enfatiza que las habilidades sociales promueven la cooperación entre personas distintas. Dicho de otra forma, el centro educativo debería construir relaciones de colaboración entre equipos multidisciplinarios para atender la diversidad en el aula como principio pedagógico para desarrollar el aprendizaje del estudiantado.

Diversos estudios centran al profesorado como objeto de investigación con el propósito de conocer su pensamiento y actuar docente (López, Echeita y Martín, 2009; López y Blanco, 2010). En este sentido, Mellado y Chaucono (2015) afirman que ahondar en el accionar pedagógico permitiría dilucidar los marcos de referencia que subyacen en el discurso docente. Es decir, explorar en las percepciones del formador o formadora ayudaría a comprender su forma de pensar y desempeño profesional.

Numerosas investigaciones han evidenciado el carácter subjetivo de las percepciones docentes, construidas en el contexto social, ancladas según componentes afectivos y valorativos (Contreras, 2010; Rojas, 2014). Prieto (2008) plantea la necesidad de explicitar las representaciones mentales y reflexionar en torno a ellas, con el propósito de modificar el pensamiento del profesorado, a través de la discusión con pares y la oportunidad de contrastar sus ideas con referentes teóricos. Es decir, las percepciones coexisten en las estructuras cognitivas del sujeto docente y se despliegan en las acciones y decisiones pedagógicas (Mellado y Chaucono, 2016).

Varios autores han sostenido que estas percepciones son representaciones implícitas que se construyen en torno a los conocimientos, experiencias y formación (López, Echeita y Martín, 2009; Ossa, 2006). Igualmente, Woolfolk, Davis y Pape (2006) sostienen que pueden movilizarse las creencias, en función de las posibilidades de interacción con pares profesionales que poseen planteamientos teóricos y diferentes argumentos. En consecuencia, resulta imperativo que los centros escolares aborden sistemáticamente las percepciones de educación inclusiva que posee el profesorado, en forma colectiva, mediante diálogos pedagógicos reflexivos para transformar su clase en aulas inclusivas.

\section{Metodología}

Es un estudio de caso de alcance descriptivo que utiliza un enfoque de investigación mixta (Hernández, Fernández y Baptista, 2010).

\subsection{Participantes}

Esta investigación presenta una muestra de 36 profesionales que se desempeñan en educación, se seleccionó de manera intencionada no probabilística, pertenecientes al equipo PIE y al profesorado de aula, de un centro escolar de la región de Los Ríos, Chile. Dicha muestra se compone de 10 educadoras diferenciales, 5 profesionales de apoyo PIE (dos fonoaudiólogos, un kinesiólogo, un psicopedagogo y un psicólogo) y 21 docentes de educación primaria y secundaria. Respecto a las personas participantes, el 86\% corresponde a mujeres y un $14 \%$ a hombres. Sus edades fluctúan entre 24 a 45 años. 


\subsection{Técnicas de recolección de información}

En este estudio, se utilizó la encuesta y entrevista como técnicas de recolección de información.

En primer lugar, se aplica el cuestionario tipo escala Likert, con el objetivo develar las percepciones sobre la práctica de educación inclusiva que posee el equipo de profesionales de la educación. Este instrumento fue validado por el equipo de investigación, tiene una validez de contenido por juicios de sujetos expertos y se analizó su fiabilidad total-elementos por cada dimensión, a través del coeficiente Alpha de Cronbach, con un valor de .97. Este proceso de validez del instrumento otorga rigurosidad científica a los datos obtenidos en este estudio. El cuestionario tiene en total 28 ítems, se estructura en cuatro dimensiones a) pedagógico-didáctica, b) aprendizaje cooperativo, c) actuación ética y d) social, las cuales se describen en la tabla 1.

Tabla 1

Descripciones de las dimensiones de educación inclusiva que posee el instrumento

\begin{tabular}{lcl}
\hline $\begin{array}{l}\text { Dimensión de } \\
\text { educación inclusiva }\end{array}$ & $\begin{array}{l}\text { Cantidad de } \\
\text { ítems }\end{array}$ & Descripción \\
\hline Pedagógico- didáctica & 8 & $\begin{array}{l}\text { Se entiende como las tareas que debería realizar el profeso- } \\
\text { rado para valorar la diversidad existente en el aula: la plani- } \\
\text { ficación y las actividades diversas de ensenanza, adaptadas } \\
\text { según las necesidades del estudiantado. }\end{array}$ \\
\hline Aprendizaje cooperativo & 9 & $\begin{array}{l}\text { Se entiende como el trabajo que tendrían que realizar el pro- } \\
\text { fesorado con el equipo de integración educativa, con el fin de } \\
\text { promover instancias que permitan generar espacios de reflex- } \\
\text { ión sobre el uso de las metodologías de trabajo más apropiado } \\
\text { para desarrollar el aprendizaje de la totalidad de sus estudi- } \\
\text { antes. }\end{array}$ \\
\hline Actuación ética & & $\begin{array}{l}\text { Es la actitud que asume el profesorado frente a estudiantes } \\
\text { que presentan alguna necesidad educativa especial, al propi- } \\
\text { ciar ambientes que favorezcan el desarrollo del aprendizaje } \\
\text { de todos los niños y niñas por igual. }\end{array}$ \\
\hline Social & 6 & $\begin{array}{l}\text { Determina la necesidad de que exista una relación de confi- } \\
\text { anza entre estudiante, familia y docente, para distinguir las } \\
\text { situaciones que requieren un trabajo colaborativo entre los } \\
\text { distintos agentes involucrados. }\end{array}$ \\
\hline
\end{tabular}

Nota: Dimensiones y descripciones de educación inclusiva basadas en Fernández, 2012.

En segundo lugar, se utilizó la entrevista semiestructurada. La información se recogió a través de los registros de audio. Se realizó un análisis de contenido con foco en las dimensiones del cuestionario. En total se desarrollaron 36 entrevistas de 40 minutos cada una, se alcanzó un total de 1440 minutos, distribuidos entre 36 profesionales durante tres meses.

A continuación, en la tabla 2 se muestran algunas preguntas orientadoras que conforman el guión de la entrevista por dimensión. 
Tabla 2

Estructura del guión de la entrevista según dimensiones de educación inclusiva

\begin{tabular}{|c|c|}
\hline Dimensión de educación inclusiva & Preguntas del guion de la entrevista \\
\hline Pedagógico- didáctica & $\begin{array}{l}\text { ¿En qué sentido las diversas estrategias de enseñanza aprendizaje } \\
\text { que trabaja en el aula están diseñadas en función de las NEE del } \\
\text { estudiantado? }\end{array}$ \\
\hline Aprendizaje cooperativo & $\begin{array}{l}\text { ¿De qué manera el trabajo cooperativo favorece la educación } \\
\text { inclusiva? }\end{array}$ \\
\hline Actuación ética & $\begin{array}{l}\text { ¿Cómo afecta la actitud del personal docente el logro de una edu- } \\
\text { cación inclusiva? }\end{array}$ \\
\hline Social & $\begin{array}{l}\text { ¿Explique la importancia de tener una relación de confianza entre } \\
\text { estudiante con NEE, su familia y demás profesionales del cuerpo } \\
\text { docente? }\end{array}$ \\
\hline
\end{tabular}

Nota: Elaboración propia 2016.

\subsection{Procedimiento de análisis}

En primer lugar, se realiza un riguroso análisis de la información obtenida por los sujetos participantes sobre la percepción de la práctica inclusiva que desarrollan en su centro escolar. Se ordenan los datos en una planilla Excel para cada dimensión de educación inclusiva. Luego se calculan las puntuación promedio por dimensión pedagógico - didáctica, aprendizaje cooperativo, actuación ética y social.

En segundo lugar, se procede a la transcripción de las entrevistas. Posteriormente, se utilizó el análisis de contenido con foco en las dimensiones de educación inclusiva: pedagógico - didáctica, aprendizaje cooperativo, actuación ética y social. De estas dimensiones surgen categorías que se analizan y ejemplifican con citas textuales para profundizar en la comprensión de las percepciones sobre la práctica de educación inclusiva que tienen docentes de aula y profesionales del Programa de Integración Escolar. El sistema utilizado para identificar las textualidades se ha organizado como sigue: Educadoras diferenciales, se utiliza las siglas $E D$, con numeración secuenciada hasta la $E D$ 10; profesionales de apoyo, se representa por la sigla $P A$ 11, en secuencia hasta los $P A 15$; docentes de educación primaria, se registrarán con la sigla Profesor EB 16, seguidamente hasta Profesor EB 24; para docentes de educación secundaria, se utilizará la sigla Profesor EM 25, seguidamente hasta Profesor EM 36.

\section{Resultados}

En primer lugar, se presentan los resultados tendientes a develar las percepciones que tienen sobre la práctica de educación inclusiva los sujetos participantes del estudio, según las dimensiones pedagógico - didáctica, aprendizaje cooperativo, actuación ética y social que considera el instrumento. En segundo lugar, se describen los hallazgos de percepción en relación con la práctica de educación inclusiva para cada dimensión y sus respectivas categorías, provenientes del análisis de contenido de las entrevistas realizadas. 


\subsection{Percepciones sobre la práctica de educación inclusiva del profesorado}

A continuación, la figura 1 presenta los resultados de las percepciones de las prácticas de inclusión que tienen las personas participantes del estudio, según dimensiones de educación inclusiva.

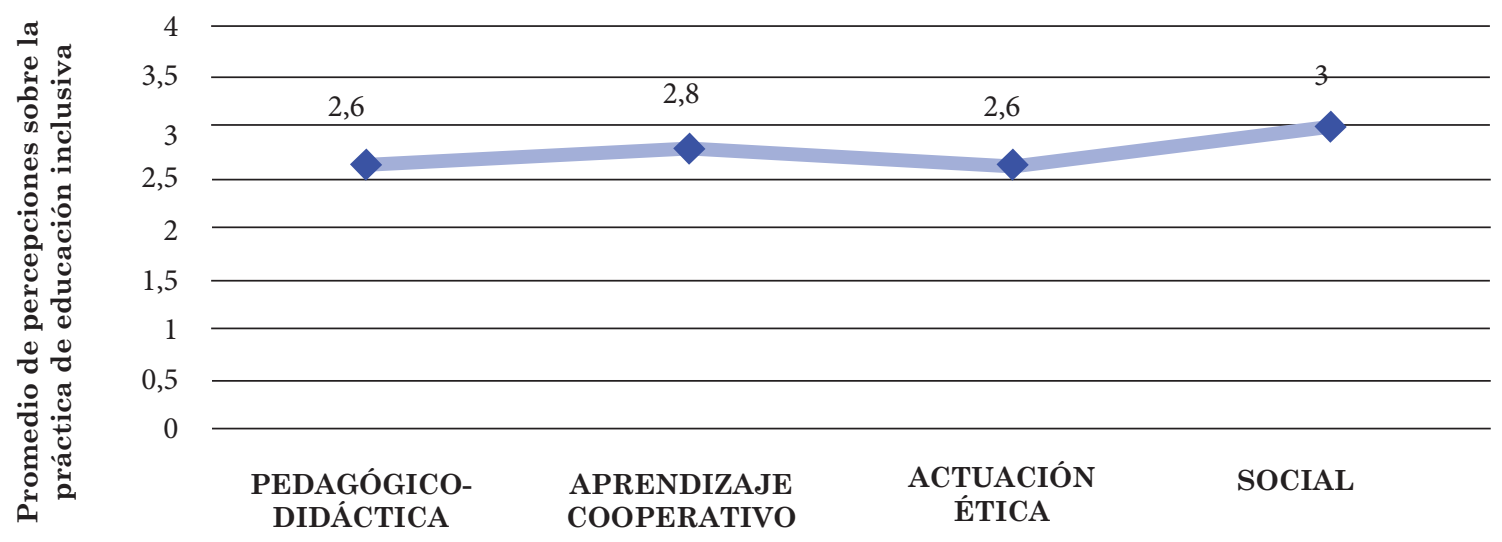

Dimensiones de educación inclusiva

Figura 1. Medias obtenidas por dimensión del cuestionario. Elaboración propia (2016).

En relación con el análisis e interpretación de los datos de la figura 1, los grupos profesionales de la educación manifiestan percepciones que tienden a ser similares entre las dimensiones de educación inclusiva. Obtienen una puntuación promedio de 2,6 en las dimensiones pedagógicas- didácticas y ética. En cambio, en la dimensión aprendizaje cooperativo alcanzan un promedio de 2,8 y de 3 en la dimensión social. Por tanto, los sujetos participantes tienen una percepción de las prácticas de educación inclusiva de su colegio que alcanza una leve tendencia positiva hacia planteamientos teóricos de inclusión.

\subsection{Hallazgos de la percepción de la práctica, según dimensiones de educación inclusiva}

\subsubsection{Dimensión pedagógico - didáctica}

Categoría: Percepciones pedagógicas segregadas

La mayoría de las personas entrevistadas poseen percepciones pedagógico- didácticas excluyentes que están centradas en la segregación de estudiantes con necesidades educativas especiales. Estas se caracterizan por obviar la atención a la diversidad en el aula.

A continuación, se presentan algunas respuestas que fueron recurrentes frente a la pregunta ¿En qué sentido las diversas estrategias de enseñanza aprendizaje que trabajas en el aula están diseñadas en función de las NEE del estudiantado? 
"Yo diseño las actividades según los contenidos que debo reforzar en clases para apoyar a estudiantes con NEE en las materias que presentan más dificultades porque no quiero que se queden retrasados respecto de sus compañeros" (Educadora Diferencial, ED2).

"Me gusta preparar diversas actividades para mis clases, que generalmente descargo de internet, se trata de llevar cuestiones novedosas para trabajar con estudiantes que presentan necesidades educativas especiales" (Profesor, EB 20).

"Yo trato de preparar guías con actividades de resolución de problemas, le muestro luego a la Educadora Diferencial, para que ella me entregue orientaciones y apoyo en clases, pero he observado su escaso dominio en el contenido de matemática, por tanto, tengo poco ayuda" (Profesor, EM 32).

Los ejemplos de las citas reflejan que los cuerpos profesionales de la educación poseen percepciones pedagógicas que restringen la inclusión en el aula. Se manifiesta una escasa variedad de estrategias para preparar buenas prácticas inclusivas, existe un desconocimiento de las adecuaciones didácticas que necesita cada estudiante para desarrollar un buen aprendizaje.

\subsubsection{Dimensión: Aprendizaje cooperativo}

Categoría: Aprendizaje individual

La mayoría de las personas entrevistadas piensa que el aprendizaje se construye de forma individual y desconoce las potencialidades del trabajo cooperativo como estrategia de educación inclusiva. Se presentan algunas percepciones que fueron recurrentes frente a la pregunta: ¿De qué manera el trabajo cooperativo favorece la educación inclusiva?

"La coordinadora PIE precisa que el estudiantado con NEE debe recibir apoyo en sala regular, pero debemos centrarnos $100 \%$ en sus necesidades, para que puedan avanzar a su propio ritmo, por eso el profesorado de las disciplinas se concentra en los demás niños y niñas. Si bien planificamos la clase en conjunto, luego en la sala no logramos hacer codocencia" (Educadora Diferencial, ED 6).

"Yo pienso que el aprendizaje cooperativo provoca en el estudiantado con NEE dispersión de las actividades desarrolladas en clases, además, se genera desorden que afecta el clima de aula que se transforma en distractor del aprendizaje" (Profesor, EM 31).

"La interacción entre estudiantes a veces favorece la inclusión porque les permite jugar y divertirse sobretodo en horarios de recreo. Pero en la sala de clases debemos diferenciar las exigencias en las actividades y la evaluación para el estudiantado con NEE, deben hacer tareas más fáciles y ser evaluados con menor exigencia" (Profesor, EB 17). 
La mayoría de profesionales que participan de este estudio tiene dificultades para implementar el aprendizaje cooperativo como una estrategia esencial para desarrollar una educación inclusiva entre los miembros de la comunidad escolar.

\subsubsection{Dimensión: Actuación ética}

Categoría: Actitud desfavorable hacia la inclusión

La mayoría de las personas entrevistadas manifiestan bajas expectativas sobre estudiantes con N.E.E y una actitud desfavorable hacia la educación inclusiva. Esta percepción del profesorado se transforma en un obstaculizador para el desarrollo de la inclusión y evidencia su escasa responsabilidad ética.

A continuación, se presentan percepciones recurrentes para la pregunta ¿Cómo afecta la actitud del personal docente el logro de una educación inclusiva?

"Yo pienso que hay que tener mucha vocación para trabajar con estos estudiantes, porque exigen una sobrecarga laboral y me complica dedicarles más tiempo, porque atraso al resto del grupo" (Profesor, EM 27).

"Estoy seguro que el cuerpo estudiantil con NEE con todas las políticas que existen en la actualidad avanzan en aspectos de socialización, pero de ahí a lograr mejorar sus aprendizajes, es bastante difícil, dado el escaso tiempo para preparar materiales $y$ además desconocemos estrategias útiles, porque nosotros no somos especialistas" (Profesor, EB 22).

"Yo me encargo de apoyar a los niños con necesidades educativas especiales, realizando actividades que involucren la estimulación de la motricidad en mis clases, ya que son más fáciles para ellos, porque no tienen las mismas capacidades" (Profesor, EB 18).

El profesorado, en su mayoría, posee actitudes negativas y escasas expectativas frente al cuerpo estudiantil con NEE, manifiesta percepciones excluyentes en sus relatos sobre las oportunidades de aprendizaje de sus estudiantes. Se observa una actitud pasiva del personal docente frente a las demandas de las personas con NEE, que demuestra un bajo sentido y responsabilidad ética de la profesión.

\subsubsection{Dimensión: Social}

Categoría: Clima de desconfianza para relacionarse

La mayoría de los discursos asociados a esta dimensión social tiende a develar que estos grupos profesionales evidencian un clima de desconfianza para relacionarse con el estudiantado con NEE. Las percepciones del equipo docente dan cuenta de las escasas relaciones de cercanía y confianza entre la comunidad docente y el cuerpo estudiantil, lo que trae consigo oportunidades restringidas para desarrollar una educación inclusiva en el centro educativo. 
A continuación, se presentan percepciones recurrentes de educación inclusiva para la pregunta: ¿Explique la importancia de tener una relación de confianza entre estudiante con NEE, su familia y demás profesionales del cuerpo docente?

"Los estudiantes que presentan necesidades educativas especiales, demandan mucho tiempo para el proceso de aprendizaje y les cuesta comunicarse de manera fluida con el equipo de profesionales. Por eso, hay pocas oportunidades para preocuparse de establecer lazos de confianza porque debemos trabajar los contenidos del currículum y en qué momento nos dedicamos a desarrollar relaciones empáticas con el grupo de estudiantes integrados" (Educadora Diferencial, ED 5).

"Yo no tengo tiempo para preocuparme de trabajar y mantener relaciones de confianza y apego con esos estudiantes, nosotros estamos contratados para lograr los objetivos de aprendizaje que se exigen a nivel curricular, tampoco me atrevo exigirles mucho porque luego aparece la familia o los profesionales PIE a reclamar" (Profesor, EM 26).

Se considera indispensable el desarrollo de habilidades sociales entre el grupo de estudiantes, sus familias y profesionales que conforman la comunidad educativa para que pueda existir confianza y seguridad en las relaciones que establecen en favor del aprendizaje de todos los niños y niñas con NEE.

\section{Conclusiones}

En concordancia con el objetivo y con base en el análisis de los resultados, se extraen algunas conclusiones preliminares que corresponden solo a este estudio.

Para la dimensión pedagógico- didáctica de educación inclusiva se puede concluir que el equipo docente tiende a segregar recurrentemente al alumnado que presenta alguna necesidad educativa especial. Esta segregación manifiesta escasas estrategias didácticas, lo que provoca una débil interacción pedagógica, considerada necesaria para desarrollar un aprendizaje significativo. En particular, predominan prácticas que dificultan el proceso enseñanza aprendizaje del estudiantado con NEE; ello se evidencia en una omisión de la educación inclusiva en sus prácticas de aula.

Para la dimensión aprendizaje cooperativo, predominan estrategias de enseñanza homogéneas e individuales en desmedro de generar actividades de aprendizaje desafiantes para todos los niños y niñas. A modo de conclusión, se pude afirmar que el equipo docente desconoce la potencialidad de los métodos cooperativos como estrategias esenciales para desarrollar una educación inclusiva en la comunidad escolar.

En relación con el sentido ético que manifiesta el equipo docente, en su mayoría excluye a estudiantes con NEE, y promueve, en el aula, solamente habilidades básicas del pensamiento, que limitan las oportunidades de aprendizaje para todos los niños y niñas. Esta actitud se torna desfavorable hacia el desarrollo de una educación inclusiva. 
En la dimensión social, los grupos profesionales de la educación demuestran un clima de desconfianza para relacionarse con el estudiantado con NEE. Esta percepción limita las oportunidades para desarrollar una educación inclusiva en el centro educativo. En este contexto, se vuelve indispensable desarrollas instancias de reflexión en la comunidad escolar, con el propósito de generar lazos de confianza que favorezcan los procesos inclusivos en los colegios.

Finalmente, este estudio permite afirmar que cuando tenemos en cuenta las diferentes necesidades y ofrecemos a todas las personas lo mismo, no hacemos más que ignorar la diversidad y generamos aún más desigualdad. Esto nos lleva, necesariamente, a buscar las herramientas que den la oportunidad de participar a todos los individuos, de desarrollar capacidades comunes por diferentes vías; de lo contrario, estaremos favoreciendo a unas personas sobre otras.

\section{Referencias}

Ainscow, M. (2009). Desarrollando sistemas de educación inclusiva. ¿Cómo podemos hacer progresar las políticas? Revista Latinoamericana de Educación Inclusiva, 17, 23-35.

Arnaiz, P. (2011). Luchando contra la exclusión: Buenas prácticas y éxito escolar. Revista Innovación Educativa, 21, 23-35.

Arnaiz, P. (2012). Escuelas eficaces e inclusivas: Cómo favorecer su desarrollo. Revista Educación Siglo XXI, 1(30), 25-44.

Arnaiz, P. (2003). Educación Inclusiva: Escuela para todos. España: Ediciones Aljibe.

Blanco, R. (2006). La atención a la diversidad en el aula y las adaptaciones del currículo. Desarrollo psicológico y educación. Madrid: Editorial Alianza Psicología.

Blanco, R. (2010). El derecho a la educación. Presentación. Revista Latinoamericana de Educación inclusiva, 4(2), 15-22.

Contreras, S. A. (2010). Las creencias y actuaciones curriculares de los profesores de ciencias de secundaria de Chile (Tesis doctoral). Universidad Complutense de Madrid, Madrid, España). Recuperada de http://eprints.ucm.es/11624/1/T32362.pdf

Duran, D. y Giné, C. (2011). La formación del profesorado para la educación inclusiva: Un proceso de desarrollo profesional y de mejora de los centros para atender a la diversidad. Revista Latinoamericana de Inclusión Educativa, 5(2), 153-170. 
Echeita, G. (2008). Inclusión y exclusión educativa. "Voz y quebranto". Revista Iberoamericana sobre Calidad, Eficacia y Cambio en Educación, 6(2), 9-18.

Fernández, J. (2012). ¿Cómo se dan los derechos educativos de los niños, niñas y adolescentes con discapacidad en el Perú?: Estudio cualitativo en Huancavelica y Villa el Salvador. Foro Educativo. Lima, Perú.

Fernández, J. (2013). Competencias docentes y educación inclusiva. Revista Electrónica de Investigación Educativa,15(2), 83-99. Recuperado de http://redie.uabc.mx/redie/article/ view/445/61

Ferrer, G. y Martínez, S. (2005). Formación de les families en el marc de l'escola inclusiva: Un repte per a les comunitats d'aprenentatge. Revista Educar, 35, 71-85.

Forteza, D. (2010). La formación del profesorado en y para la educación inclusiva desde la perspectiva de la convergencia europea. Recuperado de http://www. centrodocumentaciondown.com/uploads/documentos/36b7c169f84273904d06ad1eac3523 b10aed83f9.pdf

González, M. (2008). Diversidad e inclusión educativa: Algunas reflexiones sobre el liderazgo en el centro escolar. Revista Electrónica Iberoamericana sobre Calidad Eficacia y Cambio en Educación, 6(2), 82-99. Recuperado de http://www.rinace.net/arts/vol6num2/art7.pdf

Hernández, R., Fernández, C. y Baptista, M. (2010). Metodología de la investigación (5ª ed.). México: McGraw-Hill.

López, J. y Blanco, F. (2010). Una aproximación a las prácticas cualitativas en psicología desde una perspectiva integradora. Revista Papeles del Psicólogo, 31(1), 131-142.

López, J., Echeita, G. y Martín, E. (2009). Concepciones sobre el proceso de inclusión educativa de alumnos con discapacidad intelectual en la educación secundaria obligatoria. Revista Cultura y Educación, 21(4), 485-496.

Mellado, M. y Chaucono, J. (2015). Creencias pedagógicas del profesorado de una escuela rural en el contexto mapuche. Revista Actualidad Investigativa en Educación, 3(15), 1-19. Recuperado de http://revistas.ucr.ac.cr/index.php/aie/article/view/20924

Mellado, M. y Chaucono, J. (2016). Liderazgo pedagógico para reestructurar creencias docentes y mejorar prácticas de aula en contexto mapuche. Revista Electrónica Educare, 20(1), 1-18. Recuperado de http://www.scielo.sa.cr/pdf/ree/v20n1/1409-4258-ree-20-01-00371.pdf 
MINEDUC (2012). Informe de la comisión de expertos de educación especial. Nueva perspectiva y visión de la educación especial. Comisión de expertos de Educación Especial. Santiago, Chile.

Moriña, A. (2004). Teoría y práctica de la educación inclusiva. Archidona: Aljibe.

Murrillo, J. (2006). Una dirección escolar para el cambio: Del liderazgo transformacional al liderazgo distribuido. Revista Electrónica Iberoamericana sobre Calidad, Eficacia y Cambio en Educación, 4(4), 11-24. Recuperado de http://www.redalyc.org/articulo. oa? id $=55140403$

Ossa, C. (2006). Factores que estructuran el rol del psicólogo en educación especial. Revista de Psicología de la Universidad de Chile, 15 (2), 131-146. Recuperado de http://www. redalyc.org/pdf/264/26415208.pdf

Prieto, M. (2008). Creencias de los profesores sobre evaluación y efectos incidentales. Revista de Pedagogía, 29(84), 123-144.

Rojas, M. T. (2014). Las creencias docentes: Delimitación del concepto y propuesta para la investigación. Revista Electrónica Diálogos Educativos, 14 (27), 89-112. Recuperado de http://www.dialogoseducativos.cl/revistas/n27/rojas

Sancho, C. (2013). Propuestas inclusivas de mejora desde un enfoque metodológico y organizacional. Revista Latinoamericana de Educación Inclusiva, 1(7), 135-149.

UNESCO. (2003). Superar la exclusión mediante planteamientos integradores en la educación. Un desafío, una visión. Documento conceptual. Sección de la primera infancia y educación integradora. División de Educación Básica. París, Francia. Recuperado de http://unesdoc.unesco.org/images/0013/001347/134785s.pdf

UNESCO. (2004). Una educación de calidad para todos los jóvenes. Ginebra. 47ª Conferencia Internacional de Educación de la UNESCO.

UNESCO. (2009). Directrices sobre politicas de inclusión en la educación. Recuperado de http://unesdoc.unesco.org/images/0017/001778/177849s.pdf

Woolfolk, A., Davis, H. y Pape, S. (2006). Teacher knowledge and beliefs. En Alexander y P. Winne (Eds.), Handbook of Educational Psychology (pp. 715-737). Mahwah, N.J.: Lawrence Erlbaum Associates. 